\title{
BeaconPass: A Location Based APP Game for Traveler
}

\author{
Tsung-Yuan Ho ${ }^{1}$, Chien-Hsu Chen ${ }^{1(\bowtie)}$, Sheng-Fen Chien ${ }^{2}$, Yi-Hsuan Chen ${ }^{1}$, \\ $\mathrm{Su}-\mathrm{Yu} \mathrm{Liu}^{1}$, and Juan Sebastian Bayona ${ }^{2}$ \\ ${ }^{1}$ Department of Industrial Design, National Cheng Kung University, Tainan, Taiwan \\ \{ap313620, huokzv, suyu77121\} @gmail.com, \\ chenhsu@mail.ncku.edu.tw \\ ${ }^{2}$ Institute of Creative Industry Design, National Cheng Kung University, Tainan, Taiwan \\ schien@mail.ncku.edu.tw, sebasbayona@gmail.com
}

\begin{abstract}
BeaconPass is a smartphone/tablet application inspired by shared problems among travelers. Following our previews research; lack of internet access, GPS inaccuracy, battery life and insufficient site-specific information, reflect on travelers getting lost and missing on their touring expectations. Thus it was decided that the application's goal is to narrow the gap between previously planned activities and the exploration of a city. Beacon technology was selected as the means, from which the application would develop, to ease the exploration of a city. Given the potential that beacon technology holds for showcasing a wide offer of visiting alternatives, on a site-specific basis, the application has been packaged into a game that seeks to encourage the traveler to meet unplanned locations. Graphically, the game uses a "pirate's journey" metaphor that allows the user to level up while engaging in an open exploration of the city.
\end{abstract}

Keywords: Ibeacon · Location based game · APP · Traveler · Service design · Mobile application

\section{Introduction}

Within its planning dimension the application allows the user to design a trip by choosing desired visiting locations and setting a time and a start point from which the trip is going to develop into a complete traveling schedule. It was observed that inter-net is a recurrent source for travelers seeking guides and information regarding traveling tips. Also, social networks become a mean for validation of found information and play a role on gathering personal recommendations.

As a result of these practices, travelers tend to accumulate several desired visiting locations that applicationear unarticulated to the physical contexts that allocate them. For this reason, the application helps the process by suggesting alternative destinations relative to the places that have been chosen, allowing the user to fix the order in which the visit is going to be conducted under proximity based organization. As a result of this activity, the user will have created a visiting plan that can be shared on social networks in join the trip. 
The exploration dimension of the application starts when a previously conceived plan is set to action, providing site-specific information such as: directions, relative site information and schedule (among other), in an attempt to reduce the gap be-tween previously planned activities and unplanned situations that might applicationear along the exploration phase. This feature of the application is also designed to integrate different activities that are intrinsic to traveling practices (taking pictures, getting lost, exploring, finding food, sharing the experience, etc.) and encourages the traveler to find unplanned locations or scenes by their demand, or just randomly choosing their next stop.

\section{Literature Review}

\subsection{Location-Based Games}

These new game type take place normal virtual game's action in some dimension [1]:

- Players interacting object from simulators producing events to true events in real worlds.

- Player join the game themselves in location-based game instead of avatars and other characters interacting with each other.

- Player doing riddle and puzzle in virtual games, and chase some object or area in real world in location-based game.

- Players generating information in digital form associated with physical objects.

We using Google Scholar on March 20th, 2014 returned 483 publications. This is a full text index, so the publications found may have these keywords anywhere in their body, thus more thorough search in their content is needed. We view about 10 games in this result and make a list to observe and categorize them.

\section{- Area occupied games}

These games goal is occupy or collect some real-world's area virtually to gain score and keep some award, the service provider usually use these goal to reach their objection such like user data collection or crowdsource the area's information. For example:

- Team-It: Each player is assigned a number of skills; they have to use their own skill to rescue the virtual people in given city as much as you can [2].

- Urbanopoly: Player can occupy some spot by record "venues" picture, sold it and trade it to build a monopoly area [3].

- Frequency 1550: Players are told that they can earn citizenship in Amsterdam by collecting as many of the required 366 so-called Days of Burghership as possible. These 366 points refer to the medieval year-and-a-day rule, which is how long you had to be living inside the city walls to earn citizenship rights. Groups of four students each - two located at the headquarters at the Waag, the other two walking the streets of Amsterdam - are randomly assigned the identity of beggars or merchants, who have - as in the Middle Ages - a different social status in the game, causing merchants to win a confrontation in the city [4]. 


\section{- Treasure hunting games}

These games goal is finding several objectives in a given area, once you found these objectives; you can get some virtual award or collection. This type of action can persuade users unconsciously, and change user behavior by go to their unknown area. For example:

- iDetective: Players try to makes searching out a photo shooting location from one photograph, and go to the place where photograph was taken [5].

- See It: players use ambiguous visual clues in the form of images and video clips to find locations containing a hidden container. Players can also create and hide game content in order to help promote long-term engagement and an increasing numbers of players [6].

\section{- Learning games}

These game usually is a guide to learning something in given area, The services are tend to add some virtual data at some specific area, when player trigger the function in this area, a surrounding information context will pop up on your device. These application usually used in museum or area guide, and package it like a game to persuade user to use it. For example:

- Foursquare: automatically provide contextually relevant content in the world's major cities, user can learn local language when they entered some educational area [7].

- Tidy city: players need to physically explore their city by interpreting clues to find the correct target destination. And enable historical riddle provider walk around the city and collecting notes, images and GPS data for potential riddles [8].

\subsection{Key Technologies in Location-Based Games}

\section{- Locating people}

To get the location of the user there are several methods that can be used. The most common is to use the Global Positioning System (GPS) on smartphones as this is known to be more accurate than other methods. These include the WiFi-based positioning system (WPS) from Skyhook and Global System for Mobile Communications (GSM) based positioning. In $2010 \sim 2012$ 's mobile location-based games, most of them are using GPS for their game's location technology. At that time, iBeacon and NFC technology are still not wide use in Location-based service game.

But there are some location-based game start using the ibeacon and NFC to build there system, such like CES 2014, CNET company use ibeacon technology to ask user in CES download their application to find hidden treasure in the exhibition. By the iBeacon supported devices increasing, that will be a potential indoor-location-based game technology [9]. 


\section{- Storing and accessing data}

Once the location has been established the application needs to use this to provide information about the location in the game. This data can be sourced in many ways. Two examples of these are: using open linked data or crowdsourced data.

Open linked data is when data sets are produced and are then made freely available to all over the internet. It must also be free of all copyright and in a format that is not owned by anybody. Linked data is when data is published in a structured logical manner so that different data sets can be easily combined as they are in the same format and it is easily queried.

Crowdsourcing is using the collective knowledge of a large group of users to decide on facts. If contributors can be kept involved crowdsourced data sets can grow rapidly. It is a good way of pooling the resources of a collection of people especially for mundane tasks. It is easier for lots of people to do a little bit each than for one person to do a lot as they will get bored. However this can only be claimed to have a validation rate of about $40 \%$.

Some treasure hunting game and learning game will use crowdsourcing to ask user to help service to collect big amount of data or generate the new treasure for found. Such like Urbanopoly or Tidy city.

Finally, for the fair of the competition games, area-occupied games usually use static context, and usually unable end user to change their data or add game content.

\section{User Studies}

The development of the application started with a field recognition on the assigned perimeter within the city of Tainan, Taiwan. From our observation exercise, the commercial and touristic attributes of the neighborhood caught our attention, reason for which a semi-structured interview was designed to bring light to our project. Tourists had been decided as our point of departure based on the analysis of our previous application oxidation in which their recurrence along the streets presented an opportunity for further research. This led us to raise questions regarding the ways in which tourists engage their touristic experience, how they organize their trips and how they make sense of spatial relations while exploring the city. Based on the information collected, activities surrounding tourist undertakings were identified and separated into "Planning" and "Visiting" as major umbrella categories under which, related activities were organized using POEMS [10]. This allowed us to get a better image of how tourists were conducting their experience and what other elements were involved in each of the major activities.

To put this information under perspective, activity theory [11] served as a tool to highlight the contradictions between planning and exploring in touristic endeavors; issues such as internet access, GPS, landmarks, language barriers, touristic offer and unplanned events, built into our project's attempt to diminish the above mention gap between planning and exploration (Fig. 1). 

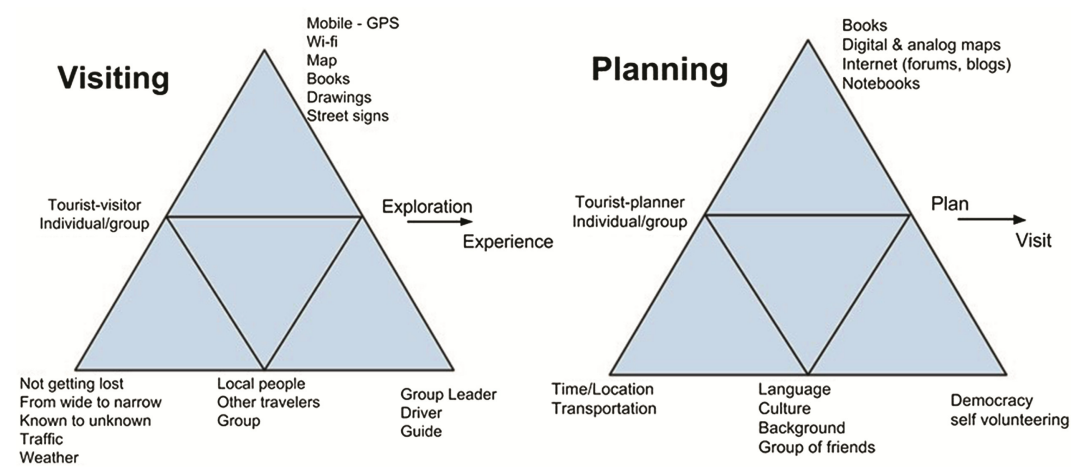

Fig. 1. Planning and visiting activity diagrams

After several discussions and fast prototyping alternatives to the identified problems, our project had developed into a location based application that would guide our user to easily reach a preset listing of desired visiting locations. The application posed the use of Beacon technology as an alternative to the technological issues that had been previously identified, also it seek to allow and encourage the user's exploration of the surroundings. Nevertheless the design of our application was still on a conceptual stage, reason for which, five personas were designed as a mean to put our application under perspective, realizing how different target customers were to use our application and how its succeeded or failed to satisfy their needs. Each of them was followed by a case scenario in which our application was to help them achieve a determined goal. From there the design of our project took off, and each of the required steps in order to complete the task was designed so that we then had a comprehensive product (Fig. 2).

\section{Andy's scenario}

\begin{tabular}{|c|c|c|c|c|c|}
\hline & Planning & $\begin{array}{l}\text { Sailing to next } \\
\text { stop }\end{array}$ & $\begin{array}{c}\text { Developing new } \\
\text { spot }\end{array}$ & Get lost & Fill the diary \\
\hline \multicolumn{6}{|l|}{ Internet } \\
\hline Mobile Phone & 1. road & & & 3. spot & \\
\hline \multicolumn{6}{|l|}{ Digital Camera } \\
\hline \multicolumn{6}{|l|}{$\begin{array}{l}\text { iBeacon of Ma- } \\
\text { pping }\end{array}$} \\
\hline \multicolumn{6}{|l|}{ iBeacon of Spot } \\
\hline iBeacon of Shop & & & & & \\
\hline
\end{tabular}

Fig. 2. Actor Andy's scenario

Adding to it, a follow up evaluation was done by abstracting each of the touch points within the personas' journey, meaning, instances in which our application eared as a tool to solve a need or mediate our users' actions. This exercise reflected on fixing navigation problems within our application by defining shortcuts and coherent pathways. 


\section{Game Design}

\subsection{Structure}

Because our Application allow users to upload their interesting photos in the trip, and also the user can share his/her traveling plans with friends, the first way for people to know this Applications through some attractive sharing on the social network sites like FB. The second way to gain more users is through the shops that cooperate with us. We will set our "islands" (which has iBeacon inside it) in the counter of the shop. If a traveler enter in that shop and see the island, he/she may download our Application. After download the Application, the first screen of the loading applications should represent the pirates; portray an image that displays the ultimate goal of the pirate development on an action that could also relate to the function of the application. The game is designed for one player, in this sense he/she will play against the system or himself in order to grow within the application. There is only the role of the pirate, nevertheless this pirate will have many possible applications, in this way the player develops its own role or personality of the pirate. It is important to have a wide range of possible combinations and also place specific characteristics. In this way one user might "compete" by showing off the uniqueness of his character in relation to his traveling experiences. The use of the application in its planning or exploration modes reflects on points that will enable the user to access to more items for customization of the pirate (Fig. 3).

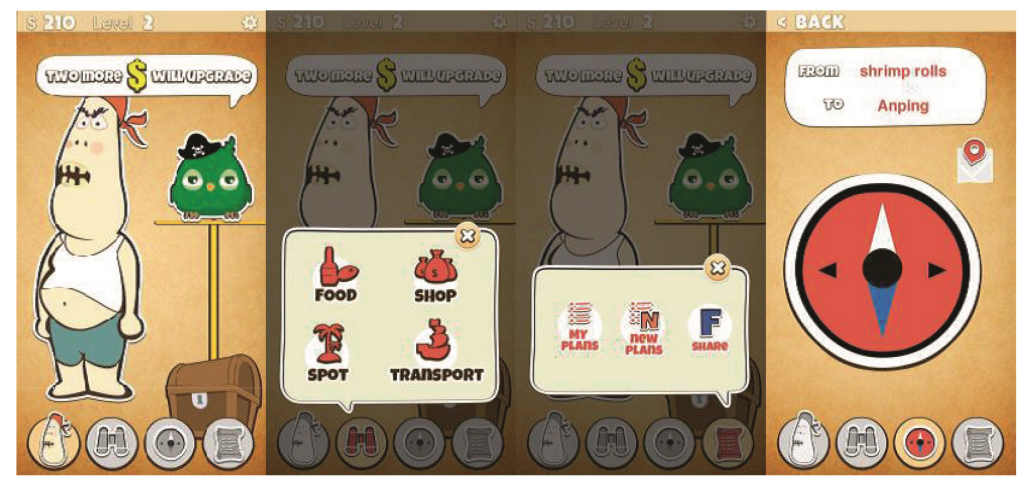

Fig. 3. Gameplays Screen

Users can customize and upgrade their own pirate by getting as many points as they can. Points are correlated to walked distances and fully accomplished plans. We encourage users to challenge their selves by setting an exploration plan (player de-fined) and find unplanned places along your way to collect as many points as you can finish your plans to upgrade. An exploration component, besides of the self-unplanned exploration, could be triggered by hiding treasures in our beacon islands setting in the whole city. In this game, gold (points) is presented as a currency within the game that is obtained by distances explored and can be traded for items. Stars represent levels of the pirate 
that will disclose different sets of purchasable items. Users will have their own inventory to save items like clothes, accessories, weapons and attributes.

\subsection{Dramatic Elements}

Traveling is an end for itself, nevertheless the way one person travels is subject to be modified, we challenge our players to engage in an exploration of their traveling spots by offering site specific opportunities of meeting unplanned locations. Our game is primarily chance based, although a preset plan might have been created by the user, the game encourages him to pursue unplanned visits, this is chance-based by the islands installed around the players' location and by the treasure alerts that might application near on the way. In this way our user falls in the category of the explorer player but also has a collector character in his pursue for character improvement. Our premise for exploration/tourism is done with the metaphor of pirates and their wondrous imaginary. In this sense our users start from a basic level or "lubber" (people not a sailor) growing with experience to become a professional traveler "Sea dog" (an experienced seaman), or a cap' $n$ (captain). The story behind the game is the traveling experience of our user, for this reason our pirates should reflect on the user's story. This is done by providing site-related items that are inspired by the local culture. This allows that different vacations will result on different character possibilities that embody the stories behind them and that trigger the memory of our users.

The world of our game is the real world itself, as the character is just a personification of our user. This is made more evident on our integrated pirate to pictures option, allowing our users to add their pirates to pictures taken, giving them a world to exist in.

\section{Prototype Implementation}

\subsection{Function Declaration}

Our Core design is play on visiting and traveling. So we build the service in iOS application service cause it can able to mobile use and it develop difficulty is much easier than Android system for iBeacon support. The functions "Display surrounding shop/ service/spot information at a stop." Is our core value in our LBS service, and it is based on several demands and restrictions inherent to exploration activities that can be answered when the application is implemented. Finally we select the iBeacon as our main LBS technology cause it has the longest sensor range. It's Also pair less, so we can receive a lot of information from different sensor at once.

\subsection{Information Architecture}

By spreading iBeacons in whole city, our mobile service can able to get the store and coordinate information by access nearest iBeacon, and merge with iOS's com-pass information and goal information in database, and display compass information-point the goal direction and distance. It will be used for "Get Lost" function and "Seek Island" 
function. Because our value is no WIFI reliable, and iBeacon must decode in client applications. That means we should build-in a large local database for map the iBeacon and Context or pre-download context when user has Internet access. The database store below data store coordination, store information, map coordinate information to map the real world's iBeacon spot to virtual map in mo-bile, it can be renewed by online database when user connect to Wi-Fi to ensure user can get the newest city information. We'll use the MySQL/PHP to implement online map server. It will combine the google map service and storage the map pin and re-late iBeacon mechanic unique ID, and it can be downloaded to client. Because of personal data, we should combine the account to share the plan, record the virtual character grow and upload the image. We can use a simple identified system or com-bine with Facebook.

We compose the "Trilateration positioning" and "Fingerprint positioning" in our method to get more accuracy location data. The localization technique used for positioning with wireless access points is based on measuring the intensity of the received signal (received signal strength in English RSS) and the method of "fingerprinting". Typical parameters useful to geo-locating the Wi-Fi hotspot or wireless access point include the SSID and the MAC address of the access point. The accuracy depends on the number of positions that have been entered into the database. The possible signal fluctuations that may occur can in-crease errors and inaccuracies in the path of the user. To minimize fluctuations in the received signal, there are certain techniques that can be application lied to filter the noise. In geometry, trilateration is the process of determining absolute or relative location points by measurement of distances, using the geometry of circles, spheres or triangles. In addition to its interest as a geometric problem, trilateration does have practical applications in surveying and navigation, including global positioning systems (GPS). In contrast to triangulation, it does not involve the measurement of angles. In two-dimensional geometry, it is known that if a point lies on two circles, then the circle centers and the two radii provide sufficient information to narrow the possible locations down to two. Additional information may narrow the possibilities down to one unique location.

We use fingerprint positioning method to measure the inaccuracy position in the city and use trilateration positioning by sensing multiple iBeacon and get RSSI for each beacons to get more accuracy position.

\section{Evaluation}

In this section, through one-on-one testing, we ask one subject to have a simple test of Beaconpass Application's paper prototype directly, and we recorded the problems he met in the test. After the game test, we had a brief interview with the subject, requesting for problems and suggestions during the game. Participant is two 23 years old student with design background. During the playing, one of the observers write down the problems he met, another one took respond for taking pictures and changing the pages of the interface for the user.

First in the preparation stage, we explain to the subject which Beaconpass applications a navigational application designed for serving outsiders or foreigners while 
traveling with pirate story as its content. We also set a task for him: suppose you are a stranger, travel to Tainan and using Beaconpass Application. During the process, you might get lost and need to plan your journey, and finally you can complete the task and upgrade your pirate. Through the observation of user testing, we could list out problems we found, and then arranged the result and the suggestions from our user with four aspects (four questions) in analysts of the Walkthrough proceeds.

\section{Conclusion}

After this project, we think the whole solution about foreigner taking trip to Tai-nan's Problem, and package in a game and user can solve these solution and take a trip in playing this application, feel yourself like a pirate. There are some part our team think we can improve in our games design. First, we can combine more Tainan's local element to fit the title "Tainan LBS games". Tainan is an ancient city and it has lots of culture element like sword lion, local sneak and ancient building in this city. Because our service is toward foreign tourist, we choose the element that foreign tourist will yearn for -pirate. And drop the element of Tainan. Although we can copy this business model to whole world cause we didn't use too much Tainan's local element, we still have to add some local element to make user more involved in Tainan City. Maybe make pirate wearing the local culture clothes is a good idea.

IBeacons are turned into islands that are installed inside local business in order to set a network of broadcasters. The islands are incorporated to our business model and also play with the pirate narrative and the larger brand construction. From our last public presentation in Tainan we received positive feedback, and good ideas were gathered from the public e.g., auto-sorting spots to minimize traveling distances when user managing locations, integrating local cultural elements into the app.

\section{References}

1. De Souza e Silva, A., Delacruz, G.: Hybrid reality games reframed: Potential uses in educational contexts. Games Cult. 1(3), 231-251 (2006)

2. Frazier, S.: Location-Based Game Platform for Behavioral Data Collection in Disaster Rescue Scenarios (2012)

3. Celino, I.: Urbanopoly - a social and location-based game with a purpose to crowdsource your urban data. In: Privacy, Security, Risk and Trust (PASSAT), 2012 International Conference on and 2012 International Conference on Social Computing (2012)

4. Avouris, N.: A review of mobile location-based games for learning across physical and virtual spaces. J. UCS 18(15), 2120-2142 (2012)

5. Yoshii, A.: A location based game to persuade users unconsciously. In: Embedded and RealTime Computing Systems and Applications (RTCSA), 2011 IEEE 17th International Conference on, vol. 1, pp. 115-120, IEEE (2011)

6. Neustaedter, C.: See it: a scalable location-based game for promoting physical activity. In: Proceedings of the ACM 2012 conference on Computer Supported Cooperative Work Companion. ACM (2012) 
7. Edge, D., Searle, E., Chiu, K., Zhao, J., Landay, J.A.: MicroMandarin: mobile language learning in context. In: Proceedings of the SIGCHI Conference on Human Factors in Computing Systems, ACM (2011)

8. Wetzel, R.: Tidy city: a location-based game supported by in-situ and web-based authoring tools to enable user-created content. In: Proceedings of the International Conference on the Foundations of Digital Games. ACM (2012)

9. Daniel Terdiman.: At CES, a hunt for hidden treasure, (2014). http://www.cnet.com/ news/at-ces-a-hunt-for-hidden-treasure/

10. Kumar, V., Whitney, P.: Daily life, not markets: customer-centered design. J. Bus. Strat. 28(4), 46-58 (2007)

11. Uden, L.: Activity theory for designing mobile learning. Mobile Learn. Organ. 1(1), 81-102 (2007)

12. Brugnoli, G.: Connecting the dots of user experience. J. Inf. Architect. 1(1), 6-15 (2009)

13. Clatworthy, S., Service innovation through touch-points: development of an innovation toolkit for the first stages of new service development. Int. J. Des. 5(1) (2011) 